\title{
Prevalência de sintomas depressivos em idosos atendidos em unidades de saúde da família e fatores associados
}

Prevalence of depressive symptoms in the elderly people assisted at the family health units and associated factors

Prevalencia de síntomas depresivos em adultos mayores atendidos en unidades de salud de la familia y factores asociados

\section{Kelly Thais Pestana Bespalhuk ${ }^{\mathrm{I}}$, Luana Vieira Coelho Ferreira' ${ }^{\mathrm{II}}$, Priscila Aguiar Mendes ${ }^{\mathrm{III}}$ Annelita Almeida Oliveira Reiners ${ }^{\text {Iv }}$, Rosemeiry Capriata de Souza Azevedov Ana Carolina Macri Gaspar Vendramini ${ }^{\mathrm{VI}}$}

\begin{abstract}
Resumo: Objetivo: analisar a prevalência de sintomas depressivos em idosos atendidos nas Unidades de Saúde da Família e fatores associados. Método: estudo transversal, realizado como recorte de uma pesquisa executada em 2015 com amostra probabilística estratificada proporcional de 557 idosos, atendidos em Unidades de Saúde da Família em Tangará da Serra. As variáveis independentes são sociodemográficas e condições de saúde. A dependente é sintomas depressivos e foi avaliada por meio da escala de depressão. Para verificar os fatores associados foi realizado regressão de Poisson. Resultados: a prevalência de sintomas depressivos foi $22,8 \%$. As variáveis associadas foram: percepção regular/ruim/péssima de saúde ( $\mathrm{RP}=6,69 ; \mathrm{IC}: 3,78-11,82)$; dependência funcional $(\mathrm{RP}=2,99$; IC: 1,85-4,83) e não ter trabalho ( RP = 3,96; IC: 1,02-15,38). Conclusão: a prevalência de sintomas depressivos foi maior que em outras pesquisas. Fatores sociais e relacionados às condições de saúde dos idosos associam-se com sintomas depressivos, sendo importante desenvolver ações para promover o envelhecimento ativo e saudável.
\end{abstract}

Descritores: Idoso; Prevalência; Depressão; Saúde Mental; Geriatria

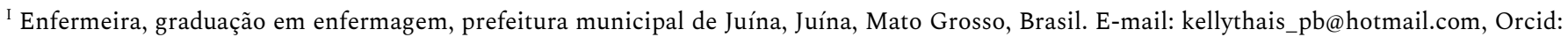
https://orcid.org/0000-0003-4394-8734.

II Enfermeira, Especialista em auditoria nos serviços de saúde, Universidade do Estado de Mato Grosso, campus de Tangará da serra, Docente auxiliar, Tangará da Serra, Mato Grosso, Brasil. E-mail: enf.luanacoelhoferreira@gmail.com, Orcid: https://orcid.org/0000-0001-6374-6246.

III Enfermeira, Mestre em enfermagem, Universidade do Estado de Mato Grosso, campus de Tangará da serra, Docente interina, Tangará da Serra, Mato Grosso, Brasil. E-mail: prih.mendes@gmail.com, Orcid: https://orcid.org/0000-0002-0752-091X.

IV Enfermeira, Doutora, Universidade Federal do Mato Grosso, Faculdade de Enfermagem, Docente adjunta, Cuiabá, Mato Grosso, Brasil. Email: annereiners.ar@gmail.com, Orcid: https://orcid.org/0000-0002-5699-8215.

V Enfermeira, Doutora, Universidade Federal do Mato Grosso, Faculdade de Enfermagem, Docente adjunta, Cuiabá, Mato Grosso, Brasil. Email: rosemeirycapriataazevedo@gmail.com, Orcid: https://orcid.org/0000-0001-7986-5768.

VI Enfermeira, Mestre em enfermagem, Universidade do Estado de Mato Grosso, campus de Tangará da serra, Docente assistente, Tangará da Serra, Mato Grosso, Brasil. E-mail: anacarolinamacri@hotmail.com, Orcid: https://orcid.org/0000-0001-5712-7115.
} 
Abstract: Objective: to analyze the prevalence of depressive symptoms in elderly people assisted at Family Health Units and associated factors. Method: This is a cross-sectional study carried out with a clipping of research in 2015 with a proportional stratified probabilistic sample of 557 elderly people assisted at Family Health Units in Tangará da Serra. The independent variables are sociodemographic and health conditions. The dependent variable is depressive symptoms and it was assessed using the depression scale. Poisson regression verified the associated factors. Results: the prevalence of depressive symptoms was $22.8 \%$. The associated variables were: regular/bad/terrible health perception ( $\mathrm{PR}=6.69$; $\mathrm{CI}$ : 3.78-11.82); functional dependence ( $\mathrm{PR}=2.99$; $\mathrm{CI}$ : $1.85-4.83$ ) and not having a job ( $\mathrm{PR}=3.96$; $\mathrm{CI}$ : 1.02-15.38). Conclusion: the prevalence of depressive symptoms was higher than in other studies. Social factors and related to the health conditions of the elderly person are associated with depressive symptoms, and it is important to develop actions to promote active and healthy aging.

Descriptors: Aged; Prevalence; Depression; Mental Health; Geriatrics

Resumen: Objetivo: analizar la prevalencia de síntomas depresivos en adultos mayores atendidos en las Unidades de Salud de la Familia y factores asociados. Método: estudio transversal, realizado como recorte de una investigación ejecutada en 2015 con una muestra probabilística estratificada proporcional de 557 adultos mayores, atendidos en Unidades de Salud de la Familia en Tangará da Serra. Las variables independentes son sociodemográficas y condiciones de salud. La variable dependiente es síntomas depresivos y fue evaluada por medio de la escala de depresión. Para verificar los factores asociados fue realizado regresión de Poisson. Resultados: la prevalencia de síntomas depresivos fue 22,8\%. Las variables asociadas fueron: percepción regular/mala/pésima de salud ( $\mathrm{RP}=6,69$; IC: $3,78-11,82)$; dependencia funcional ( $\mathrm{RP}=2,99$; IC: $1,85-4,83)$ y no tener trabajo ( $\mathrm{RP}=3,96$; IC: 1,02-15,38). Conclusión: la prevalencia de síntomas depresivos fue mayor que en otras investigaciones. Factores sociales y relacionados a las condiciones de salud de los adultos mayores se asocian con síntomas depresivos, siendo importante desarrollar acciones para promover el envejecimiento activo y saludable.

Descriptores: Anciano; Prevalencia; Depresión; Salud Mental; Geriatría

\section{Introdução}

O envelhecimento populacional é evidente no mundo e resulta na mudança do perfil demográfico e epidemiológico dos países. Durante o processo de envelhecer, o idoso apresenta alterações biológicas e psicossociais, ${ }^{1}$ bem como, diminuição progressiva da reserva funcional, ${ }^{2}$ podendo ocasionar problemas de saúde que contribuem para o desenvolvimento da depressão.

A depressão consiste em um transtorno mental de múltiplas causas, que interfere significativamente na vida social, profissional e na saúde do idoso. Por ser relacionada à área afetiva ou de humor e causar baixo autoestima situacional, é confundida com outras doenças, até mesmo com a tristeza. ${ }^{3}$ Esta condição promove graves consequências na vida do idoso, como, sofrimento psíquico, prejuízos nas atividades de vida diárias (AVD), aumento nos gastos 
3 | Bespalhuk KTP, Ferreira LVC, Mendes PA, Reiners AAO, Azevedo RCS, Vendramini ACMG

em saúde, prejuízo nos relacionamentos interpessoais, além da própria incapacidade provocada pela doença. ${ }^{4-7}$ Também é considerada um transtorno mental, o qual vem apresentando alto índice na população idosa, ${ }^{3,8}$ com maior prevalência em mulheres, ${ }^{9-13}$ pessoas com outras comorbidades ou institucionalizadas, demonstrando a necessidade da identificação e tratamento precoce desta patologia, devido as desordens médicas e neurológicas que comprometem também a qualidade de vida dos idosos. ${ }^{12}$

Pesquisas desenvolvidas no Brasil revelam que a prevalência de sintomas depressivos em idosos pode variar. Estudos desenvolvidos no Rio Grande do Sul, encontraram prevalências entre $15,2 \%{ }^{14}$ e $18,0 \% .^{15}$ Em Minas Gerais a prevalência encontrada foi de $22,0 \%{ }^{16}$ e na Bahia $52,6 \%$ dos idosos participantes em um Centro de convivências apresentavam esses sintomas. ${ }^{17}$ Essas diferenças podem ser em virtude de diversos fatores ou características das populações estudadas, demonstrando a importância de investigar este fenômeno em localidades distintas a fim de que ações de saúde possam ser planejadas de acordo com as necessidades locais.

Assim, a literatura aponta que algumas características sociodemográficas e condições de saúde são associadas a maior prevalência de sintomas depressivos nesta população, por exemplo, o sexo feminino, a baixa situação econômica, não ter trabalho, a inatividade física, a autoavaliação de saúde negativa, a incapacidade funcional, ${ }^{14-15}$ a aposentadoria, o histórico de problemas cardíacos, a insatisfação com a vida, ${ }^{15}$ e idade entre 60 e 70 anos. ${ }^{16}$

Ainda que seja uma doença muito prevalente nesta população, a literatura aponta que ela é subnotificada e muitas vezes não tratada pelos profissionais de saúde, principalmente os da atenção primária. Muitos profissionais de saúde desconhecem os métodos de rastreio, são despreparados para identificar os sintomas, como também, os associam ao próprio envelhecimento. ${ }^{17}$ Neste sentido, por ser uma doença com alta prevalência e consequências físicas e psicológicas, ela é considerada um problema de saúde pública. Com o aumento exponencial do envelhecimento populacional e as mudanças no perfil epidemiológico, há 
necessidade de investigações que possam auxiliar no conhecimento sobre prevalência, fatores envolvidos, métodos de rastreio, detecção e medidas preventivas sobre depressão em idosos.

Ademais, em estudo de revisão sistemática constatou-se que pesquisas têm sido realizadas no Brasil para avaliar a prevalência desse evento na população idosa, porém, concentram-se na região sudeste e sul, ${ }^{18}$ demonstrando a necessidade em realizar novas investigações sobre este fenômeno no centro oeste, principalmente no interior do estado de Mato Grosso (MT), pois são incipientes. Assim, conhecer as características e os fatores que estão associados à depressão em idosos podem auxiliar no planejamento de intervenções efetivas para reabilitação dessas pessoas ou para prevenção deste agravo, principalmente na atenção primária à saúde.

Assim, questiona-se: Qual a prevalência de sintomas depressivos em idosos atendidos em unidades de saúde da família (USF) em um município do médio norte do estado de Mato Grosso? Quais características sociodemográficas e de saúde estão associadas a esse evento? Acredita-se que a prevalência de sintomas depressivos em idosos seja alta e que haverá associação deste evento com alguns fatores sociodemográficos e condições de saúde. Neste sentido, o objetivo deste estudo é analisar a prevalência de sintomas depressivos em idosos atendidos nas USF e os fatores associados.

\section{Método}

Trata-se de um estudo transversal realizado como um recorte de um banco de dados de uma pesquisa primária, ${ }^{19}$ realizada em 2015, com amostra probabilística estratificada proporcional de 557 idosos atendidos em USF em Tangará da Serra, MT. A população constituiu-se em 5096 idosos identificados por meio de levantamento do cadastro das famílias nas microáreas em dez unidades básicas de saúde do município referido. 
5 | Bespalhuk KTP, Ferreira LVC, Mendes PA, Reiners AAO, Azevedo RCS, Vendramini ACMG

O cálculo da amostra foi determinado pela fórmula de população finita, atribuído intervalo de confiança de 95\%, nível de significância de 5\%, erro máximo permitido de 4\%, percentagem do fenômeno 50\%. Os idosos foram selecionados de maneira aleatória e aqueles que não atendiam aos critérios de inclusão, foram substituídos por meio de um novo sorteio até que ao final fosse possível atingir a amostra necessária. Foram incluídos no estudo pessoas de idade igual ou superior a 60 anos, atendidos nas USF e residentes em área urbana. Foram excluídos aqueles que apresentaram, no recrutamento, déficit cognitivo evidenciado pela aplicação do Mini Exame de Estado Mental, adotando o ponto de corte de acordo com o nível de escolaridade. ${ }^{20}$

A coleta de dados da pesquisa foi realizada em 2015, na residência dos idosos, com entrevista aplicando questionário sobre características sociodemográficas, condições de saúde e escalas validadas, como: Escala de Depressão Geriátrica abreviada (Geriatric Depression Scale GDS-15) para identificar os sintomas depressivos e Index de Barthel, para avaliação da capacidade funcional dos idosos. ${ }^{19}$ Os dados coletados foram duplamente digitados para correção de discordâncias e criado um banco de dados com diversas variáveis relacionadas às condições de saúde e características dos participantes com auxílio do programa Excel ${ }^{\circledR}$ (Office 2019).

Em 2017, para construção deste recorte, foi realizado acesso ao banco de dados da pesquisa primária, sendo extraídas as variáveis de interesse para este estudo, para posteriormente realizar a análise dos dados. A variável dependente deste estudo é sintomas depressivos. Para sua classificação foi utilizado o escore registrado no banco de dados obtido por meio da aplicação da GDS de cada participante, sendo considerado positivo aqueles que apresentaram valor superior a 5 pontos. ${ }^{20}$ Esta escala é um instrumento utilizado para identificar ou confirmar a suspeita de sintomas depressivos em idosos. Estudo indica que ela apresenta boa confiabilidade na detecção de casos de depressão em idosos. ${ }^{21}$

Esta, por sua vez, consiste em uma avaliação rápida composta de um questionário com 15 perguntas de respostas objetivas ( $\mathrm{sim} /$ não) a respeito de como o idoso tem se sentido durante a 
Prevalência de sintomas depressivos em idosos atendidos em unidades de saúde da... $\mid 6$

última semana. Pode ser utilizada por qualquer profissional da saúde. A cada resposta negativa do idoso ao entrevistador há a soma de 1 ponto, resultando em um escore de 0 a 15 pontos. Escores entre 0 a 5 considera-se humor normal, de 6 a 10 pontos sintomas depressivos leves e pontuações maiores que 10 pontos são considerados sintomas depressivos severos. ${ }^{21}$

As variáveis independentes são as sociodemográficas (sexo, faixa etária, estado civil; anos de estudo; renda do idoso; situação ocupacional e arranjo familiar) e as condições de saúde (percepção de saúde; problema de saúde autorreferido; número de problemas de saúde; uso de medicamento; uso de tabaco; uso de bebida alcóolica; diabetes; neoplasia; problema cardiovascular e osteomuscular; hipertensão; queda nos últimos 12 meses e capacidade funcional).

Os dados foram analisados com auxílio do programa estatístico Stata ${ }^{\circledR}$ versão11.1, sendo realizada análise descritiva dos dados com frequências relativas e absolutas e análise bivariada, utilizando teste de qui-quadrado de Pearson para identificação das variáveis associadas à variável dependente, considerando significância estatística de 5\% $(p<0,05)$. Para essa análise algumas variáveis foram categorizadas a fim de tornar-se dicotômicas, são elas: faixa etária, percepção de saúde e sintomas depressivos.

Para determinar os fatores associados aos sintomas depressivos foi realizada análise de regressão de Poisson com o método stepwise forward e incluídas no modelo de regressão as variáveis que apresentaram valor de $\mathrm{p}<0,20$. Foram estimadas a prevalência e razão de prevalência (RP) brutas e ajustadas da variável sintomas depressivos e seu intervalo de confiança (IC) $95 \%$. Permaneceram no modelo final apenas as variáveis associadas à variável dependente $(\mathrm{p}<0,05)$. A pesquisa foi conduzida de acordo com os padrões éticos exigidos, atendendo às diretrizes e normas regulamentadoras de pesquisas envolvendo seres humanos. A pesquisa primária que construiu o banco de dados utilizados neste estudo, foi aprovada pelo Comitê de Ética e Pesquisa do Hospital Júlio Muller sob Parecer 921.129, em 18 de dezembro de 2014. 


\section{Resultados}

Foram avaliados 557 idosos com idade $\geq 60$ anos, a maioria tem 60 a 69 anos $(50,8 \%)$, é do sexo feminino (61,8\%), viúvo (a), solteiro (a) ou separado (a) (54,2\%) e com renda de até um salário mínimo (70,3\%). Com relação a escolaridade, a maior parte $(42,2 \%)$ é analfabeta. A maioria dos idosos é aposentada (71,3\%), recebe até um salário mínimo (70,3\%) e mora acompanhado (84,9\%) (Tabela 01).

Tabela 01 - Distribuição dos idosos atendidos nas Unidades de Saúde da Família segundo características sociodemográficas. Tangará da Serra, Mato Grosso, 2015.

\begin{tabular}{lcc}
\hline \multicolumn{1}{c}{ Variáveis } & Frequência (n) & Porcentagem (\%) \\
\hline Sexo & 344 & 061,8 \\
Feminino & 213 & 038,2 \\
Masculino & & \\
Faixa etária & 283 & 050,8 \\
$60-69$ anos & 224 & 040,2 \\
$70-79$ anos & 50 & 009,0 \\
80 anos e mais & & \\
Estado civil & 255 & 045,8 \\
Casado (a)/ união estável & 302 & 054,2 \\
Viúvo (a)/ Solteiro (a)/ Separado (a) & & \\
Anos de estudo & 235 & 042,2 \\
Nenhum & 175 & 031,4 \\
Um a três anos & 147 & 026,4 \\
4 anos ou mais & & \\
Renda do idoso em SM & & \\
Até um salário mínimo & 392 & 070,3 \\
Mais de um salário mínimo & 165 & 029,7 \\
Situação ocupacional & & \\
Aposentado & 397 & 071,3 \\
Trabalhando & 39 & 007,0 \\
Aposentado/ trabalhando & 26 & 004,7 \\
Não trabalha & 95 & 017,0 \\
Arranjo familiar & & 015,1 \\
Mora sozinho & 473,9 \\
Mora acompanhado & 557 & 100,0 \\
Total & &
\end{tabular}

* O salário mínimo (SM) vigente no período de coleta era de $\mathrm{R} \$ 788,00$.

Fonte: Banco de dados ${ }^{19}$ 
Prevalência de sintomas depressivos em idosos atendidos em unidades de saúde da... $\mid 8$

Quanto às condições de saúde, 43,9\% dos idosos autoavaliam sua saúde como regular, $54,6 \%$ referem ter um a 4 problemas de saúde, sendo que, quase a totalidade $(98,7 \%)$ referiu apresentar algum problema de saúde. A maioria utiliza medicamentos (83,3\%), não faz uso de álcool e tabaco (78,3\% e 90,5\%, respectivamente) e apresentam capacidade funcional preservada (75,6\%). As doenças prevalentes foram as cardiovasculares e osteomusculares $(72,4 \%$ e $69,7 \%$, respectivamente) e a prevalência de sintomas depressivos foi de $22,8 \%$ (Tabela 02).

Tabela 02 - Distribuição dos idosos atendidos nas Unidades de Saúde da Família segundo condições de saúde. Tangará da Serra, Mato Grosso, 2015.

\begin{tabular}{|c|c|c|}
\hline Variáveis & Frequência (n) & Porcentagem (\%) \\
\hline \multicolumn{3}{|l|}{ Percepção de saúde } \\
\hline Ótima & 54 & 09,7 \\
\hline Boa & 192 & 34,5 \\
\hline Regular & 245 & 43,9 \\
\hline Ruim & 36 & 06,5 \\
\hline Péssima & 30 & 05,4 \\
\hline \multicolumn{3}{|c|}{ Problema de saúde autorreferido } \\
\hline Possui & 550 & 98,7 \\
\hline Não possui & 7 & 01,3 \\
\hline \multicolumn{3}{|c|}{ Número de problemas de saúde } \\
\hline Nenhum & 6 & 01,1 \\
\hline Um a quatro problemas & 304 & 54,6 \\
\hline Cinco ou mais problemas & 247 & 44,3 \\
\hline \multicolumn{3}{|l|}{ Uso de medicamentos } \\
\hline Utiliza & 464 & 83,3 \\
\hline Não utiliza & 93 & 16,7 \\
\hline \multicolumn{3}{|l|}{ Uso de tabaco } \\
\hline Tabagista & 53 & 09,5 \\
\hline Não tabagista & 504 & 90,5 \\
\hline \multicolumn{3}{|l|}{ Uso de bebida alcóolica } \\
\hline Etilista & 121 & 21,7 \\
\hline Não etilista & 436 & 78,3 \\
\hline \multicolumn{3}{|l|}{ Diabetes } \\
\hline Tem diabetes & 116 & 20,8 \\
\hline Não tem diabetes & 441 & 79,2 \\
\hline \multicolumn{3}{|l|}{ Neoplasia } \\
\hline Tem neoplasia & 15 & 02,7 \\
\hline Não tem neoplasia & 542 & 97,3 \\
\hline Problema Cardiovascular & & \\
\hline
\end{tabular}


9 | Bespalhuk KTP, Ferreira LVC, Mendes PA, Reiners AAO, Azevedo RCS, Vendramini ACMG

\begin{tabular}{lcc} 
Tem & 403 & 72,4 \\
Não tem & 154 & 27,6 \\
Problema Osteomuscular & & \\
Tem & 388 & 69,7 \\
Não tem & 169 & 30,3 \\
Hipertensão & & \\
Tem & 374 & 67,2 \\
Não tem & 183 & 32,8 \\
Queda nos últimos 12 meses & & \\
Caiu & 220 & 39,5 \\
Não caiu & 337 & 60,5 \\
Capacidade funcional & & \\
Dependente & 136 & 24,4 \\
Independente & 421 & 75,6 \\
Sintomas depressivos & & \\
Ausente & 430 & 77,2 \\
Leve & 110 & 19,7 \\
Severo & 17 & 03,1 \\
\hline & 557 & 100,0 \\
\hline
\end{tabular}

Fonte: Banco de dados ${ }^{19}$

A análise bivariada entre sintomas depressivos e características sociodemográficas mostrou associação estatisticamente significativa com situação ocupacional $(\mathrm{p}=0,037)($ Tabela 03). A prevalência dos sintomas depressivos em idosos que não trabalham foi 3,96 vezes maior em relação a prevalência dos idosos que trabalham. Em idosos aposentados e idosos aposentados que trabalham, a prevalência foi 2,94 e 2,49 vezes maior, respectivamente, comparados aos idosos que trabalham.

Tabela 03 - Prevalência de sintomas depressivos dos idosos atendidos nas Unidades de Saúde da Família segundo variáveis sociodemográficas (n=127). Tangará da Serra, Mato Grosso, 2015.

\begin{tabular}{lcccc}
\hline \multicolumn{1}{c}{ Variáveis } & $\mathbf{n}$ & Prevalência (\%) & RP bruta $\left(\right.$ IC95\%) ${ }^{\boldsymbol{*}}$ & Valor de $\boldsymbol{p}^{+}$ \\
\hline Sexo & & & & \\
Feminino & 85 & 24,7 & $1,25(0,90-1,73)$ & 0,172 \\
Masculino & 42 & 19,7 & 1,0 & \\
Faixa etária & & & & \\
$60-69$ anos & 69 & 24,4 & $1,15(0,84-1,56)$ & 0,366 \\
70 anos e mais & 58 & 21,2 & 1,0 &
\end{tabular}

Estado civil 
Prevalência de sintomas depressivos em idosos atendidos em unidades de saúde da... $\mid 10$

\begin{tabular}{|c|c|c|c|c|}
\hline Viúvo/solteiro/separado & 62 & 24,3 & $1,12(0,83-1,53)$ & \multirow{2}{*}{0,434} \\
\hline Casado/união estável & 65 & 21,5 & 1,0 & \\
\hline \multicolumn{5}{|l|}{ Anos de estudo } \\
\hline Nenhum & 64 & 27,2 & $1,48(0,99-2,21)$ & \multirow[t]{3}{*}{0,093} \\
\hline Um a três anos de estudo & 36 & 20,6 & $1,12(0,71-1,75)$ & \\
\hline Quatro anos ou mais de estudo & 27 & 18,4 & 1,0 & \\
\hline \multicolumn{5}{|l|}{ Renda do idoso em SM } \\
\hline Até um SM & 8 & 25,0 & $0,90(0,48-1,68)$ & \multirow[t]{2}{*}{0,760} \\
\hline Mais de um SM & 119 & 22,7 & 1,0 & \\
\hline \multicolumn{5}{|l|}{ Situação ocupacional } \\
\hline Aposentado & 90 & 22,7 & $2,94(0,97-8,88)$ & \multirow{4}{*}{0,037} \\
\hline Trabalha & 3 & 07,7 & 1,0 & \\
\hline Aposentado e trabalha & 5 & 19,2 & $2,49(0,65-9,58)$ & \\
\hline Não trabalha & 29 & 30,5 & $3,96(1,28-12,28)$ & \\
\hline \multicolumn{5}{|l|}{ Arranjo familiar } \\
\hline Mora sozinho & 19 & 22,6 & 1,00 & \multirow{2}{*}{0,966} \\
\hline Mora acompanhado & 108 & 22,8 & $1,00(0,65-1,55)$ & \\
\hline
\end{tabular}

* Razão de prevalência (RP) e Intervalo de 95\% de confiança (IC). ${ }^{+}$Teste de associação qui-quadrado

Fonte: Banco de dados ${ }^{19}$

Quanto à associação entre sintomas depressivos e condições de saúde, verificou-se significância estatística para as seguintes variáveis: percepção de saúde regular/ruim/péssima (p $<0,001)(\mathrm{RP}=2,25 ;$ IC 95\% 1,57-3,22), cinco ou mais números de problemas de saúde $(\mathrm{p}<0,001)$ $(\mathrm{RP}=2,06 ; \mathrm{IC} 95 \% 1,50-2,83)$, problemas osteomusculares $(\mathrm{p}=0,001)(\mathrm{RP}=0,53 ; \mathrm{IC} 95 \% 0,35-0,80)$ e cardiovasculares $(\mathrm{p}=0,004)(\mathrm{RP}=1,56 ;$ IC 95\% 1,15-2,11), queda nos últimos 12 meses $(\mathrm{p}=0,002)$ $(\mathrm{RP}=1,60 ; \mathrm{IC} 95 \% 1,18-2,17)$ e dependência para capacidade funcional $(\mathrm{p}<0,001)(\mathrm{RP}=2,36$; IC $95 \%$ 1,76-3,17 (Tabela 04).

A prevalência de sintomas depressivos entre os idosos com percepção de saúde regular/ruim/péssima foi $25 \%$ maior dos que classificaram sua saúde como ótima/boa. Aqueles com cinco ou mais problemas de saúde tiveram prevalência de sintomas depressivos de 2,06 vezes mais em relação aos que apresentaram nenhum a quatro problemas de saúde. Entre os que apresentavam problemas cardiovasculares e osteomusculares, a prevalência de sintomas depressivos foi de $56 \%$ e $53 \%$, respectivamente, comparados aos que não apresentavam tais 
11 | Bespalhuk KTP, Ferreira LVC, Mendes PA, Reiners AAO, Azevedo RCS, Vendramini ACMG

condições clínicas. Em idosos que caíram nos últimos 12 meses, a prevalência foi de 60\%, comparados aos que não caíram e nos dependentes, constatou 2,36 vezes mais a prevalência dos sintomas depressivos que em independentes.

Tabela 04 - Prevalência de sintomas depressivos dos idosos atendidos nas Unidades de Saúde da Família segundo variáveis condições de saúde em idosos ( $\mathrm{n}=127)$ Tangará da Serra, Mato Grosso, 2015.

\begin{tabular}{|c|c|c|c|c|}
\hline Variáveis & $\mathrm{n}$ & Prevalência (\%) & RP bruta (IC95\%) & Valor de $p$ \\
\hline \multicolumn{5}{|l|}{ Percepção de saúde } \\
\hline Ótima/Boa/Regular & 86 & 17,5 & 1,0 & \multirow{2}{*}{$<0,001$} \\
\hline Ruim/péssima & 41 & 62,1 & $3,55(2,70-4,64)$ & \\
\hline \multicolumn{5}{|l|}{ Problema de saúde } \\
\hline $\operatorname{Sim}$ & 126 & 22,9 & $1,60(0,25-9,92)$ & \multirow{2}{*}{0,589} \\
\hline Não & 1 & 14,3 & 1,0 & \\
\hline \multicolumn{5}{|l|}{ Número de problemas de saúde } \\
\hline Nenhum a quatro problemas & 48 & 15,8 & 1,0 & \multirow{2}{*}{$<0,001$} \\
\hline Cinco ou mais problemas & 79 & 31,98 & $2,06(1,50-2,83)$ & \\
\hline \multicolumn{5}{|l|}{ Uso de medicamentos } \\
\hline $\operatorname{Sim}$ & 112 & 24,1 & $1,49(0,91-2,44)$ & \multirow{2}{*}{0,093} \\
\hline Não & 115 & 16,1 & 1,0 & \\
\hline \multicolumn{5}{|l|}{ Uso de tabaco } \\
\hline Sim & 11 & 20,8 & 1,0 & \multirow{2}{*}{0,709} \\
\hline Não & 116 & 23,0 & $1,10(0,63-1,92)$ & \\
\hline \multicolumn{5}{|l|}{ Uso de bebida alcoólica } \\
\hline $\operatorname{Sim}$ & 28 & 23,1 & $1,01(0,70-1,47)$ & \multirow{2}{*}{0,920} \\
\hline Não & 99 & 22,7 & 1,0 & \\
\hline \multicolumn{5}{|l|}{ Diabetes } \\
\hline $\operatorname{Sim}$ & 29 & 25,0 & $1,12(0,78-1,61)$ & \multirow{2}{*}{0,526} \\
\hline Não & 98 & 22,2 & 1,0 & \\
\hline \multicolumn{5}{|l|}{ Neoplasia } \\
\hline $\operatorname{Sim}$ & 2 & 13,3 & 1,0 & \multirow{2}{*}{0,376} \\
\hline Não & 125 & 23,0 & $1,72(0,47-6,35)$ & \\
\hline \multicolumn{5}{|l|}{ Problema cardiovascular } \\
\hline $\operatorname{Sim}$ & 53 & 30,3 & $1,56(1,15-2,11)$ & \multirow{2}{*}{0,004} \\
\hline Não & 74 & 19,4 & 1,0 & \\
\hline \multicolumn{5}{|l|}{ Problema osteomuscular } \\
\hline $\operatorname{Sim}$ & 103 & 26,6 & $0,53(0,35-0,80)$ & \multirow{2}{*}{0,001} \\
\hline Não & 24 & 14,2 & 1,0 & \\
\hline \multicolumn{5}{|l|}{ Hipertensão } \\
\hline $\operatorname{Sim}$ & 93 & 24,9 & $1,33(0,94-1,90)$ & \multirow{2}{*}{0,097} \\
\hline Não & 34 & 18,6 & 1,0 & \\
\hline \multicolumn{5}{|l|}{ Queda nos últimos 12 meses } \\
\hline Sim & 65 & 29,6 & $1,60(1,18-2,17)$ & 0,002 \\
\hline
\end{tabular}




$\begin{array}{lcccc}\text { Não } & 62 & 18,4 & 1,0 \\ \text { Capacidade funcional } & & & & \\ \text { Independente } & 72 & 17,1 & 1,0 & <0,001 \\ \text { Dependente } & 55 & 40,4 & 2,36(1,76-3,17) & \end{array}$

*Razão de prevalência (RP) e Intervalo de 95\% de confiança (IC). ${ }^{\dagger}$ Teste de associação qui-quadrado

Fonte: Banco de dados ${ }^{19}$

Para a análise de regressão de Poisson, foram incluídas todas as variáveis com $\mathrm{p} \leq 0,20$ na seguinte ordem: percepção de saúde, número de problemas de saúde, capacidade funcional, problema osteomuscular, queda nos últimos 12 meses, problema cardiovascular, situação ocupacional, escolaridade, uso de medicamentos, hipertensão, sexo e faixa etária.

Por meio da associação dos sintomas depressivos com as variáveis sociodemográficas e de saúde, no modelo final permaneceram com significância estatística as seguintes variáveis: percepção de saúde, capacidade funcional, situação ocupacional, ajustado por sexo e faixa etária (Tabela 05). A maior prevalência de sintomas depressivos encontrada foi entre os idosos com percepção de saúde ruim/péssima, funcionalmente dependente e que não trabalham. Foi realizado teste de bondade de ajuste, mostrando que o modelo é adequado ( $\mathrm{p}=0,4386)$.

Tabela 05 - Modelo de Regressão múltipla de Poisson: variáveis associadas aos sintomas depressivos de idosos atendidos nas Unidades de Saúde da Família. Tangará da Serra, Mato Grosso, 2015.

\begin{tabular}{lccc}
\hline \multicolumn{1}{c}{ Variáveis e categorias } & RP bruta (IC95\%) & RP ajustada (IC95\%) ${ }^{*}$ & Valor de $\boldsymbol{p}^{+}$ \\
\hline $\begin{array}{l}\text { Percepção de saúde } \\
\text { Ótima/boa/Regular }\end{array}$ & 1,0 & 1,0 & $<0,001$ \\
$\begin{array}{l}\text { Ruim/péssima } \\
\text { Capacidade funcional }\end{array}$ & $3,55(2,70-4,64)$ & $6,69(3,78-11,82)$ & \\
$\begin{array}{l}\text { Dependente } \\
\text { Independente }\end{array}$ & $2,36(1,76-3,17)$ & $2,99(1,85-4,83)$ & $<0,001$ \\
Situação ocupacional & 1,0 & 1,0 & \\
Aposentado & & & \\
Trabalha & $2,94(0,97-8,88)$ & $3,28(0,90-11,95)$ & 0,071 \\
Aposentado e trabalha & 1,0 & 1,0 & 0,141 \\
Não trabalha & $2,49(0,65-9,58)$ & $3,41(0,66-17,49)$ & $\mathbf{0 , 0 4 7}$ \\
Sexo & $3,96(1,28-12,28)$ & $3,96(1,02-15,38)$ & \\
Feminino & & & 0,665 \\
Masculino & $1,25(0,90-1,73)$ & $0,89(0,55-1,45)$ &
\end{tabular}


13 | Bespalhuk KTP, Ferreira LVC, Mendes PA, Reiners AAO, Azevedo RCS, Vendramini ACMG

\section{Faixa Etária}

60 a 69 anos

$1,15(0,84-1,56)$

$1,44(0,92-2,26)$

70 anos e mais

$1,0 \quad 1,0$

1,0

0,105

${ }^{*}$ Razão de prevalência (RP) e Intervalo de 95\% de confiança (IC). ${ }^{\dagger}$ Teste de associação qui-quadrado. ${ }^{+}$Variáveis de ajuste do modelo de Regressão de Poisson.

Independente do sexo e faixa etária, idosos com percepção de saúde ruim/péssima possui uma prevalência de sintomas depressivos 6,69 vezes maior quando comparada a prevalência de sintomas depressivos de idosos com percepção de saúde ótima/boa/regular. A prevalência de sintomas depressivos em idosos dependentes funcionalmente foi $99 \%$ maior em relação a prevalência de sintomas depressivos de idosos independentes. Em idosos que não trabalham, a prevalência de sintomas depressivos foi de 3,96 vezes mais, comparados aos idosos que trabalham independente do sexo e faixa etária.

\section{Discussão}

A prevalência de sintomas depressivos encontrada neste estudo foi de $22,8 \%$, maior que em outros estudos realizados na região sul do país. ${ }^{14-15}$ No entanto, foi semelhante a outras pesquisas na literatura, uma realizada em Minas Gerais ${ }^{16}$ e outra na Paraíba, ${ }^{22}$ cujas prevalências foram, respectivamente, $22 \%$ e $28,1 \%$. Em uma pesquisa de base populacional, realizada no Rio Grande do Sul, com 1593 idosos pertencentes a área de cobertura das Estratégias de Saúde da Família, a prevalência do evento foi de $18 \%{ }^{15}$

Estudos desenvolvidos em Minas Gerais encontraram diferentes prevalências de depressão em idosos. Um deles avaliou idosos residentes na área rural e encontrou que $22 \%$ deles apresentaram esses sintomas. ${ }^{16} \mathrm{O}$ outro, realizado com participantes hospitalizados em unidades clínicas e cirúrgicas, identificou que 39\% dos idosos apresentavam sintomas depressivos leve a moderado e $8 \%$ sintomas severos. ${ }^{23}$ 
Prevalência de sintomas depressivos em idosos atendidos em unidades de saúde da... | 14

A disparidade entre os resultados encontrados nos estudos analisados pode ser em virtude da diferença entre as populações estudadas, tanto em relação a suas características, quanto à localização do país. O Rio Grande do Sul é um dos estados com maior índice de desenvolvimento humano, maior expectativa de vida, devido a melhores condições de vida, sanitárias, científica e tecnológica e, talvez por isso, a prevalência de sintomas depressivos na população idosa nesta região do país seja menor em relação a outras. ${ }^{24}$

Além disso, idosos que residem na área rural apresentam diferentes características daqueles que vivem na área urbana, ainda mais aqueles que são atendidos pelas USF, hospitalizados, institucionalizados ou que frequentam os centros de convivência para idosos. As pesquisas que identificaram alta prevalência de sintomas depressivos em idosos atendidos pelas USF, ${ }^{15,22}$ assim como este, reforçam a importância de ações para identificação deste agravo nesta população, reabilitação da saúde mental por meio de tratamento multiprofissional, como também, ações para prevenção e promoção da saúde na atenção primária.

A literatura aponta que a depressão, muitas vezes, é negligenciada pelos profissionais de saúde, pouco investigada na atenção primária, como também pelos familiares, por ser considerada de difícil diagnóstico, ou ser classificada como algo natural do processo de envelhecimento. ${ }^{17}$ Assim, torna-se importante desenvolver educação permanente com os profissionais de saúde para capacitálos a identificar os idosos que apresentam sintomas depressivos, bem como, oferecer uma assistência integral para promoção da saúde mental desta população.

A percepção negativa de saúde associada aos sintomas depressivos também é um resultado observado em outros estudos. ${ }^{1,15,25-26}$ Idosos com percepção de saúde ruim ou muito ruim apresentam maior incidência no desenvolvimento de depressão, quando comparados aos idosos que referiam ter boa avaliação de sua saúde. ${ }^{1}$ A literatura ainda aponta que a própria percepção ruim ou muito ruim da saúde pode também ser considerada como um sintoma 
15 | Bespalhuk KTP, Ferreira LVC, Mendes PA, Reiners AAO, Azevedo RCS, Vendramini ACMG

depressivo, ${ }^{25}$ além de estar associada a presença de doenças crônicas que são patologias comuns na população idosa. ${ }^{27}$

A percepção de saúde é conceituada como sendo um dado subjetivo de como o indivíduo se vê em relação ao seu estado geral, na qual incluem dimensões biológicas, psíquica e social, que se associam a elementos da vida da pessoa (invalidez, perda cognitiva, laboral e sexual, dentre outros). ${ }^{28}$ Assim, pelo fato dos idosos deste estudo, em sua maioria, possuírem problemas de saúde, baixa escolaridade e renda, talvez possa ter contribuído para autoavaliarem sua saúde como negativa e consequentemente apresentarem sintomas depressivos.

Outro achado é a maior prevalência em idosos com dependência funcional, resultado também encontrado em outras pesquisas., ${ }^{4,9,12,14-16,25} \mathrm{O}$ comprometimento da capacidade funcional está intimamente relacionado aos sintomas depressivos, pois, à medida que o indivíduo se torna dependente para as AVD há maior acometimento emocional, desencadeando a depressão. ${ }^{4} \mathrm{~A}$ partir do momento em que o idoso diminui a vontade em realizar atividades básicas de vida, atribui-se a indicativos desses sintomas, o que dificulta, por sua vez, na execução de tais atividades. Desta forma, ocasiona a este idoso, maior grau de dependência funcional. ${ }^{14}$

A associação da dependência funcional e prevalência de sintomas depressivos faz com que o idoso tenha uma visão pessimista de sua vida, ocasionando prejuízo na vivência cotidiana. O fato de necessitar de ajuda de outra pessoa para realizar atividades de seu cotidiano, torna-se desconfortável para o idoso, e muitas vezes, leva-o a sentir-se inútil, contribuindo então para o desenvolvimento de sintomas depressivos.

Pesquisa realizada no município de Campina Grande, Paraíba, verificou associação de depressão e dependência funcional para realização de atividades instrumentais de vida diária (AIVD) e AVD e, concluíram que indivíduos sem depressão eram mais ativos. ${ }^{4}$ Assim, as incapacidades funcionais tornam-se fortes indicadores de depressão, além da institucionalização de idosos e do aparecimento de síndromes geriátricas. ${ }^{29}$ Ao se deparar com 
Prevalência de sintomas depressivos em idosos atendidos em unidades de saúde da... | 16

limitações em desempenhar atividades que requeiram maior capacidade física e cognitiva, o idoso pode sofrer alterações de humor e baixa autoestima, contribuindo para o desenvolvimento da depressão. Torna-se importante a identificação dos fatores que possam causar tais limitações, ou as possíveis modificações psicológicas para prevenir o surgimento da doença.

Neste contexto, a família da pessoa idosa assume papel importante para o reestabelecimento funcional, emocional do idoso, bem como, para o tratamento de sintomas depressivos. Contudo, o enfermeiro deve estar inserido nesse processo, contribuindo não apenas para a independência funcional do idoso, mas também para o reestabelecimento do seu vínculo familiar por meio do processo terapêutico. ${ }^{10}$

A associação com sintomas depressivos com a situação ocupacional de não trabalhar se mostrou evidente neste estudo como também na literatura. Idosos residentes em zona urbana e que não trabalham apresentam maior associação com a ocorrência desse evento, quando comparados a idosos que exercem atividade de trabalho remunerada. ${ }^{14}$ Em contrapartida, outras pesquisas apontaram associação de sintomas depressivos com idosos aposentados..$^{10,15,30}$

Idosos institucionalizados que não exercem atividades de trabalho apresentam maior insatisfação de vida devido ao fato de não possuírem autonomia financeira, corroborando para o surgimento de sintomas depressivos. ${ }^{30}$ Idosos aposentados têm grau de quadros depressivos mais elevados pelo fato de serem desvalorizados socialmente, já que, manter-se ativo no campo de trabalho ajuda o idoso a sentir-se útil na prestação de serviço para a sociedade. ${ }^{10}$

Neste contexto, idosos que não trabalham estão mais ociosos, haja vista que com a inatividade no mercado de trabalho ocorre a diminuição da renda e de aspectos relacionados a autonomia financeira, ou seja, este idoso deixa de ser o provedor financeiro, antes considerado como produtivo passa a ser dependente financeiramente, o que pode favorecer o surgimento de sintomas depressivos. 
17 | Bespalhuk KTP, Ferreira LVC, Mendes PA, Reiners AAO, Azevedo RCS, Vendramini ACMG

Neste estudo, idosos com autoavaliação ruim de saúde, dependentes funcionais e que não trabalham apresentam maiores prevalências de sintomas depressivos. Nesse sentido, a fim de reduzir a prevalência desse evento torna-se importante a necessidade de intervenções nos serviços de saúde, por meio das políticas de assistência a pessoa idosa, bem como, estabelecer terapêutica apropriada para a reabilitação da saúde mental e física nas USF dos municípios em todo o Brasil, além de trabalhar a reinserção desta população na sociedade.

Os resultados são relevantes para inferir que idosos atendidos nas USF também apresentam depressão, logo, é imprescindível que estes sejam investigados e acompanhados. Neste sentido, o Ministério da Saúde recomenda a avaliação global do idoso na atenção primária que inclui a avaliação de sintomas depressivos por meio da GDS. ${ }^{20}$ A depressão é um dos transtornos psiquiátricos mais prevalentes na população idosa, e precisa ser investigada. Assim, cabe a equipe de USF promover o acolhimento, identificar os riscos e a presença da doença e desenvolver ações para reabilitação da saúde do idoso.

Desta forma, faz-se um alerta para os serviços de políticas públicas, em especial a rede de atenção primária, na busca de investimentos em ações de promoção de saúde e capacitação dos profissionais das USF para a identificação e diagnóstico precoce da doença. Diante disso, estabelecer medidas que visem um plano terapêutico eficaz em que possa estimular o idoso para sua independência e autonomia, de modo a garantir vida digna ao idoso e propiciar melhoria da sua capacidade funcional e mental.

A limitação deste estudo se refere ao delineamento transversal, em que não é permitido avaliar causalidade e efeito. No entanto, a amostra do estudo é representativa do município e permite a extrapolação dos resultados para a população como um todo. 
Prevalência de sintomas depressivos em idosos atendidos em unidades de saúde da... $\mid 18$

\section{Conclusão}

Neste estudo, a prevalência de sintomas depressivos em idosos encontrada foi de $22,8 \%$, considerada alta em comparação com outras pesquisas da literatura. Conclui-se que aos fatores associados foram percepção regular/ruim/péssima de saúde, dependência funcional e não trabalhar. Assim, independente do sexo e faixa etária, idosos com percepção de saúde ruim/péssima, dependentes funcionais e que não trabalham possuem uma prevalência de sintomas depressivos maior.

Estes resultados são importantes uma vez que, o conhecimento desses fatores podem auxiliar os profissionais de saúde que atuam nas USF no planejamento de ações para prevenção e diagnóstico da doença, como também subsidiar a criação de políticas públicas para a promoção do envelhecimento ativo e saudável.

É importante investir na realização de pesquisas futuras com delineamento de estudos de coorte para identificação dos fatores de riscos que levam a desenvolver depressão, objetivando melhor compreensão dos fatores associados. Além destes, há importância de desenvolver estudos que avaliem medidas preventivas que possam reduzir a incidência ou progresso da doença.

\section{Referências}

1. Melo RA, Silva AKAG, Fernandes FECV, Oliveira MMA, Almeida TKP, Melo RA, et al. Sintomas depressivos em grupos de terceira idade. Rev Pesq Cuid Fundam. 2019;11(2):297-303. doi: https://doi.org/10.9789/2175-5361.2019.v11i2.297-303

2. Jobim FARC, Jobim EFC. Atividade física, nutrição e estilo de vida no envelhecimento. UNOPAR Cient Ciênc Biol Saúde (Online). 2015 [acesso em 2018 set 15];17(4):298-308. Disponível em: https://revista.pgsskroton.com/index.php/JHealthSci/article/view/3274/3005

3. World Health Organization (WHO). Depression and other common mental disorders: global health estimates. Geneva: World Health Organization; 2017.

4. Araújo GKN, Sousa RCR, Souto RQ, Silva EGJ, Eulálio MC, Alves FAP, et al. Functional capacity and depression in elderly. Rev Enferm UFPE On Line [Internet]. 2017 [cited 2018 Sept 15]; 11(10):3778-86. 
19 | Bespalhuk KTP, Ferreira LVC, Mendes PA, Reiners AAO, Azevedo RCS, Vendramini ACMG

Available from: https://periodicos.ufpe.br/revistas/revistaenfermagem/article/view/22591

5. Cheruvu VK, Chiyaka ET. Prevalence of depressive symptoms among older adults who reported medical cost as a barrier to seeking health care: findings from a nationally representative sample. BMC Geriatr. 2019;19(1):192. doi: https://doi.org/10.1186/s12877-019-1203-2

6. Grochtdreis T, Brettschneider C, Bjerregaard F, Bleich C, Boczor S, Härter M, et al. Costeffectiveness analysis of collaborative treatment of late-life depression in primary care. (GermanIMPACT). Eur Psychiatry. 2019;57:10-8. doi: https://doi.org/10.1016/j.eurpsy.2018.12.007

7. Hoell A, Weyerer S, Maier W, Wagner M, Scherer M, Stark A, et al. The impact of depressive symptoms on utilization of home care by the elderly: longitudinal results from the AgeMooDe study. J Affect Disord. 2016;204;247-54. doi: https://doi.org/10.1016/j.jad.2016.08.004

8. Smith M, Haedtke C, Shibley D. Late life depression detection: an evidence-based guideline. J Gerontol Nurs. 2015;41(2):18-25. doi: https://doi.org/10.3928/00989134-20150115-99

9. Lampert CDT, Ferreira VRT. Fatores associados à sintomatologia depressiva em idosos. Aval Psicol. 2018;17(2):205-12. doi: https://doi.org/10.15689/ap.2018.1702.14022.06

10. Magalhães JM, Carvalho AMB, Carvalho SM, Alencar DC, Moreira WC, Parente ACM. Depressão em idosos na estratégia saúde da família: uma contribuição para a atenção primária. REME Rev Min Enferm. 2016;20:e947. doi: https://doi.org/10.5935/1415-2762.20160016

11. Marques JFS, Sá SC, Freitas Filho W, Santo LRE, Prince KA, Oliveira MVM. Transtorno depressivo maior em idosos não institucionalizados atendidos em um centro de referência. Arq Ciênc Saúde. 2017;24(4):20-4. doi: https://doi.org/10.17696/2318-3691.24.4.2017.804

12. Nóbrega IP, Leal MCC, Marques APO. Prevalência de sintomas depressivos e fatores associados em idosos institucionalizados no município de Recife, Pernambuco. Estud Interdiscip Envelhec. [Internet]. 2016 [acesso em 2018 ago 20];21(2):135-54. Disponível em: https://seer.ufrgs.br/RevEnvelhecer/article/view/50346

13. Mohebbi M, Agustini B, Woods LR, McNeil JJ, Nelson MR, Shah RC, et al. Prevalence of depressive symptoms and its associated factors among healthy community-dwelling older adults living in Australia and the United States. Int J Geriatr Psychiatry. 2019;34:1208-16. doi: https://doi.org/10.1002/gps.5119

Editora Científica: Tânia Solange Bosi de Souza Magnago

Editora Associada: Rafaela Andolhe 


\section{Autor correspondente}

Ana Carolina Macri Gaspar Vendramini

E-mail: anacarolinamacri@hotmail.com

Endereço: Rua 19 A, número 1713W, Jardim Itália

CEP: $78300-000$

\section{Contribuições de Autoria}

1 - Kelly Thais Pestana Bespalhuk

Concepção ou desenho do estudo/pesquisa, análise e/ou interpretação dos dados, revisão final com participação crítica e intelectual no manuscrito.

\section{2 - Luana Vieira Coelho Ferreira}

Análise e/ou interpretação dos dados, revisão final com participação crítica e intelectual no manuscrito.

\section{3 - Priscila Aguiar Mendes}

Análise e/ou interpretação dos dados, revisão final com participação crítica e intelectual no manuscrito.

\section{4 - Annelita Almeida de Oliveira Reiners}

Revisão final com participação crítica e intelectual no manuscrito).

\section{5 - Rosemeiry Capriata de Souza Azevedo}

Contribuições: 3) revisão final com participação crítica e intelectual no manuscrito).

\section{6 - Ana Carolina Macri Gaspar Vendramini}

Concepção ou desenho do estudo/pesquisa, análise e/ou interpretação dos dados, revisão final com participação crítica e intelectual no manuscrito.

\section{Como citar este artigo}

Bespalhuk KTP, Ferreira LVC, Mendes PA, Reiners AAO, Azevedo RCS, Vendramini ACMG. Prevalência de sintomas depressivos em idosos atendidos em unidades de saúde da família e fatores associados Rev. Enferm. UFSM. 2021 [Acesso em: Ano Mês Dia]; vol.11 e34: 1-20. DOI:https://doi.org/10.5902/2179769248484 